\title{
IMPLEMENTACIÓN DE ESTRATEGIAS INTERDISCIPLINARIAS PARA EL RETORNO AL TRABAJO: REVISIÓN DE LITERATURA
}

\author{
IMPLEMENTATION OF INTERDISCIPLINARY STRATEGIES FOR RETURNING TO WORK:
} LITERATURE REVIEW

\section{Hayda Josefa Cáceres Peñaranda ${ }^{1}$, Luisa Fernanda Contreras Bethel ${ }^{2}$, Paola Andrea Garzón Cortés ${ }^{3}$, Paula Alejandra Rivera Casas ${ }^{4}$, Valery Tatiana Rozo Hernández ${ }^{5}$.}

\section{Resumen}

Objetivo: analizar las estrategias interdisciplinarias implementadas en el retorno al trabajo de trabajadores que reportan enfermedad laboral y/o accidente de trabajo.

Método: revisión de literatura que utiliza las palabras claves (tesauros): "Reubicación laboral", "Reincorporación laboral", "Trabajo", "Estrategias" y "Terapia Ocupacional", uso de boléanos AND y OR, búsqueda de la evidencia en las bases de datos: ProQuest, Ebsco, PubMed, Embase y Scopus, en el periodo 2006 - 2016.

Resultados: los hallazgos muestran una selección de 20 artículos, encontrados principalmente en la base de datos de ProQuest. La estrategia interdisciplinaria más utilizada es la identificación de la exigencia del puesto de trabajo, las habilidades del trabajador y la evaluación del WRI (Entrevista del rol del trabajador) utilizada por Terapia Ocupacional.

Conclusiones: las evaluaciones como estrategia del retorno al trabajo se centran en las capacidades y funciones físicas, con frecuencia no cuentan con el impacto psicosocial, conductual y ambiental como factores determinantes del rendimiento y capacidad laboral; es por esto que, con el uso de una evaluación basada en el desempeño ocupacional, los Terapeutas Ocupacionales lograrían evaluar de forma segura y efectiva la capacidad del trabajador para desempeñar tareas.

\section{Palabras claves:}

Estrategias, Retorno al trabajo, Terapia Ocupacional

\footnotetext{
Terapeuta ocupacional. Especialista en Salud Ocupacional de la Universidad de Cartagena convenio Universidad Francisco de Paula Santander, Formación en Integración Sensorial Fundación Vértice Colombia, Terapeuta Ocupacional Escuela Colombiana de Rehabilitación, Profesor de carrera del Programa de Terapia Ocupacional de la Escuela de Medicina y Ciencias de la Salud de la Universidad del Rosario. Número de ResearchedID: L-3931-2016, ORCID: http://orcid.org/0000-0002-4751-9227 Correo postal: Diagonal 17B \#90-53, Torre 5 Apto 903 Bogotá - Colombia Teléfono: +57 3106252928..Email: hayda.caceres@urosario.edu.co, aydacaceresp@gmail.com

Estudiante de Terapia Ocupacional Universidad del Rosario. Teléfono: +57 3012130054. Email: luisaf.contreras@urosario.edu.co, luisafernandacb94@gmail.com

Estudiante de Terapia Ocupacional Universidad del Rosario. Teléfono: +57 3203419110. Email: paolaa.garzon@urosario.edu.co

Estudiante de Terapia Ocupacional Universidad del Rosario. Teléfono: +57 3212840015. Email: paulaal.rivera@urosario.edu.co

Estudiante de Terapia Ocupacional Universidad del Rosario. Teléfono: +57 3212813562. Email: valery.rozo@urosario.edu.co, valery.ro7@gmail.com
} 


\begin{abstract}
Objective: Analyze the interdisciplinary strategies implemented in the return to work of workers who report occupational illness and / or work accident.

Method: literature review using topics as: "job relocation," "Return to work", "Work", "Strategies" and "Occupational Therapy", using boolean AND and OR, search for evidence in the databases: ProQuest, EBSCO, PubMed, Embase and Scopus, in the period 2006 - 2016 Results: the findings show a selection of 20 items, found mainly in the ProQuest database. The interdisciplinary approach most commonly used is the identification of the requirements of the job and the skills of the worker and WRI (Worker role interview) assessment used by occupational therapy.

Conclusions: assessments as return to work strategy focus on skills and physical functions, often they lack the psychosocial, behavioral and environmental impact as determinants of performance and working capacity; which is why using a web-based occupational performance evaluation, occupational therapists would achieve safely and evaluate effective worker's ability to perform tasks
\end{abstract}

\title{
Keywords:
}

Strategies, Return to work, Occupational Therapy

Recepcionado: 05/10/2016

Aceptado: 31/05/2017

\section{INTRODUCCIÓN}

Una de las ocupaciones más significativas en la que los adultos se involucran es el trabajo. Este hace parte integral de la vida del ser humano, es una ocupación que trasciende la cultura, etnia y género (McFadden MacDonald, Fogarty, Le, \& Merritt, 2010). El trabajo también promueve y satisface las necesidades fundamentales humanas tales como: solidez económica, estatus social, gestión del tiempo y socialización (Huguette A 2013). A su vez, permite que las personas fortalezcan su autodeterminación y propósito de vida, lo cual facilita de manera significativa la recuperación de la confianza en sus capacidades, en especial, cuando se presenta algún tipo de Accidente Laboral (AL) o Enfermedad Labora (EL) (Huguette A, 2013)

Según la Organización Internacional del Trabajo OIT, 2005), cada año se reportan 268 millones de AL no mortales y 160 millones de nuevos casos de EL. A nivel nacional, para el año 2015 la Federación de Aseguradores Colombianos (FASECOLDA) reportó 723.836 Accidentes aborales y 9.583 Enfermedades Laborales calificados. Por otra parte el número de casos de incapacidades permanentes-parciales (IPP) por AL fue de 10.714 y por EL fue de 4.240 (FASECOLDA, 2015)

En el 2013, Boada y Moreno realizan una investigación de tipo descriptivo conformada por doce (12) Administradoras de Riesgos Laborales (ARL) de la ciudad de Bogotá, quienes hacen un análisis de las bases de datos relacionadas con la gestión de los programas de rehabilitación integral de las ARL, en un periodo de doce
(12) meses comprendido entre mayo del 2007 a mayo de 2008. En dicho análisis se evidenció un total de 6014 casos de rehabilitación, de los cuales se identificaron 3071 casos exitosos que corresponden al 51\% de la población y 2943 (49\%) casos no exitosos. Este indicador permite detectar que la gestión en los programas de rehabilitación integral y reincorporación ocupacional aún no tiene un impacto significativo en éstos procesos.

El Manual de Procedimientos para la Rehabilitación y Reincorporación Ocupacional de los Trabajadores en el Sistema General de Riesgos Profesionales, explica:

Al interior del Sistema General de Riesgos Profesionales (SGRP), la integración SOcio-ocupacional va más allá de la ubicación/ reubicación laboral u orientación ocupacional, implica además que el trabajador tenga la capacidad de involucrarse y desempeñar los roles sociales y familiares que le correspondan; por tanto, las acciones que se dirijan al logro de este objetivo deberán estar interrelacionadas y dirigidas hacia varios frentes en forma simultánea: hacia la persona, su familia, los sectores educativo y laboral así como hacia la comunidad en general. (Ministerio de la Protección Social, 2010, p.28). 
Con lo anterior, se evidencia que los programas de retorno laboral requieren la implementación de intervenciones interdisciplinarias que involucran al trabajador y a las personas que conforman su entorno familiar, social y laboral. A su vez, estas intervenciones deben estar enfocadas a la generación de estrategias pertinentes con respecto a la participación del trabajador en los diferentes contextos, partiendo de sus capacidades y limitaciones derivadas del AL o EL; es allí, donde se ve implicada la Terapia Ocupacional, una de las disciplinas que interviene en el proceso de retorno al trabajo como lo mencionan Turner A, Foster M, y Johnson S, en su libro "Terapia Ocupacional y Disfunción Física":

El terapeuta puede ayudar a que el individuo se prepare para reincorporarse al trabajo, asimilando lo mejor posible el entorno y las expectativas laborales. Su capacidad para analizar y graduar la actividad puede ayudar a que la persona alcance el nivel necesario para desempeñar su trabajo (Turner A, Foster M, \& Johnson S, 2003)

El terapeuta ocupacional en el área laboral busca favorecer la independencia del trabajador por medio de la caracterización de factores que influyen en el proceso de adaptación al puesto de trabajo posterior a un Accidente o Enfermedad Laboral, sustentando sus procesos de evaluación desde el Modelo de la Ocupación Humana $(\mathrm{MOHO})$, el cual permite al profesional identificar y comprender las capacidades y limitaciones del individuo en las áreas de la ocupación desde los subsistemas de volición, habituación y capacidad de desempeño, que proporciona la estructuración de un concepto ocupacional y un plan de intervención acorde a las habilidades de los trabajadores, teniendo en cuenta sus intereses, hábitos, rutinas y roles (Kielhofner, 2011).

Esta revisión tiene como objetivo identificar las principales estrategias relacionadas con el retorno laboral en sus diferentes fases, con el fin de implementarlas y brindar un sustento teórico a las intervenciones que se realizan al interior de las empresas.

\section{MÉTODO}

El presente estudio se inscribe dentro de una revisión de literatura sobre las estrategias utilizadas en los procesos de reincorporación laboral, donde se plantea la siguiente pregunta de investigación: ¿Qué estrategias han sido utilizadas en los procesos de retorno laboral a nivel mundial, durante el periodo de 2006 a 2016? . Esta pregunta resulta de interés ya que conlleva a conocer que se ha investigado a nivel mundial sobre los procesos de retorno laboral, el nivel de desempeño funcional, cognitivo y psicológico de un trabajador para retornar al trabajo y la preparación que este necesita.

Para esto se determinan los tópicos de búsqueda, teniendo como criterios de inclusión: documentos tipo artículos, tesis y protocolos, trabajadores diagnosticados con enfermedad de origen común y laboral; años de publicación de 2006 a 2016. Los criterios de exclusión fueron: documentos tipo páginas web, noticias, texto completo no disponible y artículos que mencionan la reincorporación post licencia de maternidad.

La estrategia de búsqueda incluyó descriptores de Ciencias de la Salud (DeCS) en español y descriptores $(\mathrm{MeSH})$ en inglés. Se hicieron combinaciones mediante el uso de los boléanos AND y OR, y los tesauros tales como "Reubicación laboral", "Reincorporación laboral", "Trabajo", "Estrategias" y "Terapia Ocupacional".

\section{Búsqueda de evidencia}

Se inició la búsqueda de los artículos con tres combinaciones de tesauros en inglés y español las cuales se ajustan al objetivo del estudio: "Work integration" OR "Return to work" OR "Back to work" AND "Strategies" AND "Occupational Therapy"); ("Worker role interview (WRI)" AND "Occupational Therapy" AND "work"), y ("Work environment impact scale (WEIS)" AND "Occupational therapy" AND "work").

Dicha búsqueda se realizó en las bases de datos: ProQuest, Ebsco, PubMed, Embase y Scopus. Posteriormente se establecieron dos filtros que permitieron el análisis de la información. En el primer filtro se seleccionaron artículos que presentaran en el título uno o más de los tesauros escogidos, además de la lectura del abstract. El segundo filtro comprendió la lectura completa de los documentos, para finalmente detectar veinte (20) artículos en total.

\section{Análisis de la información}

Los resultados fueron registrados en una rejilla de Excel y analizados a través de las siguientes variables: estrategias usadas a nivel interdisciplinario y disciplinas que lideran estos procesos. Con dicha información se realizó la construcción del documento final. 


\section{RESULTADOS}

Con la primera combinación ("Occupational therapy") AND ("Work integration" OR "return to work" OR "back to work") AND (strategies) se encontró un (1) artículo por cada una de las bases de datos EBSCO, EMBASE y PUBMED, cuatro (4) artículos en SCOPUS y siete (7) en PROQUEST.

Con la combinación (“Worker role interview” (WRI) AND ("occupational therapy") AND (work) solo se haIlaron dos (2) artículos en la base de datos PROQUEST y tres (3) en SCOPUS.
Con la combinación ("Work environment impact scale" WEIS)) AND ("Occupational therapy") AND (Work) solo se encontró un (1) artículo en la base de datos SCOPUS. El análisis de resultados se realiza sobre los veinte (20) artículos arrojados en la búsqueda metodológica. En la tabla No 1, se observan las estrategias interdisciplinarias categorizadas, por autor y disciplina.

Tabla No 1. Estrategias Interdisciplinares en procesos de retorno al trabajo por fases.

\begin{tabular}{|l|l|l|}
\hline \multicolumn{1}{|c|}{ Estudios } & \multicolumn{1}{|c|}{ *Metodología \& Población diana } & Estrategia interdisciplinar \\
\hline $\begin{array}{l}\text { Fisker, Langberg, } \\
\text { Petersen, \& } \\
\text { Mortensen, 2013 }\end{array}$ & $\begin{array}{l}\text { *Ensayo clínico aleatorizado (ECA) } 770 \text { adultos entre } \\
\text { 18-65 años desempleados, empleados o en riesgo de } \\
\text { enfermedad, presentan dolor lumbar de un mínimo } \\
\text { de cuatro semanas y son referidos a un centro de } \\
\text { atención ambulatoria. }\end{array}$ & $\begin{array}{l}\text { Intervención multidisciplinar } \\
\text { orientada al trabajo }\end{array}$ \\
& & \\
\hline
\end{tabular}




\begin{tabular}{|c|c|c|}
\hline Descripción & Disciplina & Resultados \\
\hline $\begin{array}{l}\text { Plan de rehabilitación interdisciplinario de } 2 \\
\text { semanas basado en un enfoque biopsicosocial, } \\
\text { con intervenciones dirigidas al paciente, en áreas } \\
\text { de:-Trabajo Social: análisis de necesidades a ni- } \\
\text { vel local y municipal.-Intervención psicológica: } \\
\text { *Listado de síntomas SCL-90: mide la angustia } \\
\text { psicológica, en } 10 \text { subescalas: somatización, } \\
\text { comportamiento obsesivo-compulsivo, fobia, } \\
\text { depresión, ansiedad, hostilidad, sensibilidad in- } \\
\text { terpersonal, psicoticismo y una escala adicional } \\
\text { del sueño y el apetito.*Cuestionario de Creencias } \\
\text { de evitación del miedo (FABQ), evalúa las creen- } \\
\text { cias de los pacientes sobre cómo la actividad } \\
\text { física y el trabajo afectan su dolor.-Evaluación del } \\
\text { médico laboral: evaluación del estado actual del } \\
\text { trabajador y su capacidad para retornar al trabajo } \\
\text { (en relación a las tareas); análisis ergonómico del } \\
\text { puesto de trabajo (exploración de la disposición } \\
\text { física y la organización del lugar de trabajo. Al } \\
\text { final del proceso se realiza un informe con las re- } \\
\text { comendaciones de modificaciones en el trabajo } \\
\text { o vida diaria, sean temporales o permanentes y } \\
\text { los cambios en el estilo de trabajo o las condicio- } \\
\text { nes ergonómicas; estas se entregan al empleador } \\
\text { y al ente gubernamental municipal. }\end{array}$ & $\begin{array}{l}\text { Fisioterapia y } \\
\text { Psicología }\end{array}$ & $\begin{array}{l}\text { Facilita la reincorporación laboral a tra- } \\
\text { vés del trabajo terapéutico sea en tiempo } \\
\text { completo, tiempo parcial o con tareas } \\
\text { modificadas. El resultado principal es la } \\
\text { ausencia de enfermedad o de sintomato- } \\
\text { logía de dolor en los usuarios que partici- } \\
\text { paron en el programa, esto se evaluó } 12 \\
\text { meses después de la inclusión. Se observa } \\
\text { que cada vez aumenta más el número de } \\
\text { pacientes que han regresado a trabajar. La } \\
\text { disminución en la sintomatología de do- } \\
\text { lor revela menor proporción de casos por } \\
\text { discapacidad, mejor calidad de vida en } \\
\text { los trabajadores y ahorro en gastos para } \\
\text { la empresa y la comunidad. Se asegura } \\
\text { la puntualidad en las diferentes inter- } \\
\text { venciones., la comunicación entre todas } \\
\text { las partes interesadas, el contacto con el } \\
\text { lugar de trabajo y se logran graduar las } \\
\text { demandas del trabajo. }\end{array}$ \\
\hline
\end{tabular}




\begin{tabular}{|c|c|c|}
\hline Estudios & *Metodología \& Población diana & Estrategia interdisciplinar \\
\hline Coutu et al ,2011 & $\begin{array}{l}\text { *Diseño descriptivo de perspectiva mixta de un } \\
\text { programa de desarrollo basado en la teoría / Inter- } \\
\text { vención basada en el usuario. } 8 \text { Terapeutas ocupa- } \\
\text { cionales, } 4 \text { médicos laborales, } 4 \text { psicólogos y } 40 \\
\text { pacientes con enfermedad laboral de } 12 \text { semanas } \\
\text { debido al dolor musculoesquelético quienes pla- } \\
\text { nean iniciar un plan de rehabilitación. }\end{array}$ & $\begin{array}{l}\text { Programa de Toma de decisiones } \\
\text { compartidas (Shared Decisión } \\
\text { Making- SDM) }\end{array}$ \\
\hline Shaw et al, 2009 & $\begin{array}{l}\text { *Método de evaluación clínica en factores psicoso- } \\
\text { ciales y laborales. } 21 \text { investigadores y médicos par- } \\
\text { ticipantes de Decade of the Flags Working Group, } \\
\text { Universidad Keele, Reino Unido. }\end{array}$ & $\begin{array}{l}\text { Screening clínico para determinar } \\
\text { factores ocupacionales }\end{array}$ \\
\hline $\begin{array}{l}\text { Høgelund, Holm, } \\
\text { McIntosh, } 2010\end{array}$ & $\begin{array}{l}934 \text { trabajadores, menores de } 60 \text { años con incapa- } \\
\text { cidad mayor a } 8 \text { semanas por enfermedad. }\end{array}$ & $\begin{array}{l}\text { Método Econométrico para el } \\
\text { retorno gradual al trabajo }\end{array}$ \\
\hline Snodgrass, J. (2011). & $\begin{array}{l}\text { *Revisión basada en la literatura. } 23 \text { estudios exa- } \\
\text { minados, de los cuales: } \\
-7 \text { examinaron los efectos del ejercicio terapéutico. } \\
-3 \text { educación al usuario. }\end{array}$ & $\begin{array}{l}\text { Aplicación de principios del } \\
\text { condicionamiento operante y la } \\
\text { actividad física en el retorno al } \\
\text { trabajo }\end{array}$ \\
\hline Snodgrass, J. (2011). & $\begin{array}{l}\text { multidisciplinarios } \\
\text { - } 2 \text { efecto de agentes físicos }\end{array}$ & $\begin{array}{l}\text { Ejercicio físico para el retorno al } \\
\text { trabajo }\end{array}$ \\
\hline
\end{tabular}




\begin{tabular}{|c|c|c|}
\hline Descripción & Disciplina & Resultados \\
\hline $\begin{array}{l}\text { Proceso conjunto de la toma de decisiones entre } \\
\text { el paciente y el médico laboral, en el que se inter- } \\
\text { cambia información y expresan preferencias, lle- } \\
\text { gando a un acuerdo con respecto a los objetivos y } \\
\text { un plan de acción a seguir. }\end{array}$ & $\begin{array}{l}\text { Terapia } \\
\text { Ocupacional } \\
\text { Psicología } \\
\text { social y } \\
\text { Marco de } \\
\text { apoyo a las } \\
\text { decisiones } \\
\text { de Ottawa } \\
\text { (ODSF) }\end{array}$ & $\begin{array}{l}\text { Mejora la seguridad y efectividad en la } \\
\text { toma de decisiones, creando condiciones } \\
\text { de calidad de vida para el trabajador. Re- } \\
\text { duce la duración de la discapacidad rela- } \\
\text { cionada con el trabajo, a través de mejores } \\
\text { servicios de rehabilitación. }\end{array}$ \\
\hline $\begin{array}{l}\text { Se aplicaron cuestionarios, entrevista y visitas el } \\
\text { lugar de trabajo, se observan demandas físicas del } \\
\text { trabajo, capacidad de modificar las tareas, estrés } \\
\text { laboral, apoyo social del lugar de trabajo, la sa- } \\
\text { tisfacción laboral, la expectativa de reanudar el } \\
\text { trabajo, y el temor a una nueva lesión. Se estable- } \\
\text { cieron cinco criterios de evaluación de métodos } \\
\text { de detección: fiabilidad, rendimiento predictivo, } \\
\text { factibilidad, aceptabilidad, y la congruencia con } \\
\text { las intervenciones plausibles. }\end{array}$ & $\begin{array}{l}\text { Terapia } \\
\text { Ocupacional }\end{array}$ & $\begin{array}{l}\text { Los cuestionarios realizados al trabajador } \\
\text { representan un método muy viable y son } \\
\text { útiles si se administran de forma rutinaria, } \\
\text { además las visitas al lugar de trabajo pro- } \\
\text { porcionan una oportunidad de inspeccio- } \\
\text { nar el entorno físico de trabajo, evaluar el } \\
\text { entorno social y organizacional. }\end{array}$ \\
\hline $\begin{array}{l}\text { Durante la participación del programa, el trabaja- } \\
\text { dor inicia su retorno al trabajo con menos horas } \\
\text { laborales, pero recibe salario normal por las horas } \\
\text { trabajadas y con pago de incapacidades. Cuando } \\
\text { la salud del trabajador mejora, las horas de tra- } \\
\text { bajo se incrementan hasta que el trabajador logra } \\
\text { cumplir las horas regulares. }\end{array}$ & Economía & $\begin{array}{l}\text { Reduce la probabilidad de una nueva le- } \\
\text { sión y permite al trabajador adquirir una } \\
\text { remuneración en el tiempo de recupera- } \\
\text { ción junto con el retorno a sus labores. }\end{array}$ \\
\hline $\begin{array}{l}\text { Sesiones quincenales de una hora de acondicio- } \\
\text { namiento físico con énfasis en modificación del } \\
\text { comportamiento. }\end{array}$ & $\begin{array}{l}\text { Fisioterapia y } \\
\text { Psicología }\end{array}$ & $\begin{array}{l}\text { Disminuye la tasa de ausentismo laboral } \\
\text { a partir de la graduación de la actividad } \\
\text { física con condicionamientos en su com- } \\
\text { portamiento. }\end{array}$ \\
\hline $\begin{array}{l}\text { Realizar rutinas de ejercicio físico en personas } \\
\text { con patología lumbar }\end{array}$ & Fisioterapia & $\begin{array}{l}\text { No se encuentra suficiente evidencia de la } \\
\text { efectividad de esta estrategia y se sugiere } \\
\text { un tratamiento holístico con énfasis psico- } \\
\text { social. }\end{array}$ \\
\hline
\end{tabular}




\begin{tabular}{|c|c|c|}
\hline Estudios & *Metodología \& Población diana & Estrategia interdisciplinar \\
\hline $\begin{array}{l}\text { Hoefsmit, N., } \\
\text { Houkes, I., \& } \\
\text { Nijhuis, F. J. (2012). }\end{array}$ & $\begin{array}{l}\text { *Revisión sistemática de literaturaFueron incluidos } \\
23 \text { artículos, el objetivo del estudio es detectar e } \\
\text { identificar las características de las intervenciones } \\
\text { para el retorno al trabajo. }\end{array}$ & Formación y capacitación \\
\hline $\begin{array}{l}\text { Tschernetzki, P. } \\
2007\end{array}$ & $\begin{array}{l}\text { *Estudio de cohorte Trabajadores lesionados que } \\
\text { recibían servicios de rehabilitación en el Millard } \\
\text { Health entre } 1999 \text { y } 2005 \text {. El tamaño de la pobla- } \\
\text { ción de } 6.709 \text { fase de pre-cambio y } 6.717 \text { en la } \\
\text { fase de post-cambio. El } 70,1 \% \text { hombres y el } 29,9 \% \\
\text { eran mujeres con edad media de } 40,4 \text { años. }\end{array}$ & Programa de Salud Millard \\
\hline Huguette, A. 2013 & $\begin{array}{l}\text { *Revisión sistemática de literaturaSe identificaron } \\
\text { nueve modelos entre esos el Modelo Canadiense } \\
\text { de rendimiento ocupacional, Modelo de Ocupa- } \\
\text { ción Humana (MOHO), Persona-Ambiente-Ocupa- } \\
\text { ción rendimiento modelo] fueron seleccionados }\end{array}$ & $\begin{array}{l}\text { Evaluación del desempeño a partir } \\
\text { del Modelo de la Ocupación } \\
\text { Humana }\end{array}$ \\
\hline $\begin{array}{l}\text { Bejerholm \& } \\
\text { Areberg. } 2014 \text { y } \\
\text { Fenger, } 2009 \text { y } \\
\text { Yngve, } 2015\end{array}$ & $\begin{array}{l}\text { *Estudio transversal, investigar cómo los usuarios } \\
\text { perciben la utilidad clínica de la versión sueca del } \\
\text { (WRI-S). } 187 \text { participantes realizaron la evaluación, } \\
89 \text { habían realizado más de } 20 \text { evaluaciones, } 51 \\
\text { utilizan la prueba en la semana, } 47 \text { trabajan en } \\
\text { áreas de rehabilitación. }\end{array}$ & $\begin{array}{l}\text { Utilización de la Entrevista del rol } \\
\text { del trabajador (WRI) }\end{array}$ \\
\hline $\begin{array}{l}\text { Stergiou-kita, Yantzi, } \\
\text { \& Wan, } 2010\end{array}$ & $\begin{array}{l}\text { *Diseño de investigación cualitativa y constructi- } \\
\text { vista } 10 \text { Terapeutas ocupacionales, } 7 \text { participantes } \\
\text { para análisis de retorno al trabajo. }\end{array}$ & $\begin{array}{l}\text { Evaluación de preparación para el } \\
\text { trabajo }\end{array}$ \\
\hline
\end{tabular}




\begin{tabular}{|c|c|c|}
\hline Descripción & Disciplina & Resultados \\
\hline $\begin{array}{l}\text { Programa de formación a trabajadores en proceso } \\
\text { de rehabilitación para determinar la clasificación } \\
\text { del reintegro en: regular, asistido e individualiza- } \\
\text { do. }\end{array}$ & $\begin{array}{l}\text { Medicina } \\
\text { Laboral }\end{array}$ & $\begin{array}{l}97 \% \text { de los trabajadores retornaron a su } \\
\text { puesto de trabajo de origen. Se evidenció } \\
\text { reducción del tiempo de incapacidades } \\
\text { pasando de } 48 \text { a } 32 \text { días. }\end{array}$ \\
\hline $\begin{array}{l}\text { Se examinan factores como: persona, procesos, } \\
\text { participación del empleador, políticas, procedi- } \\
\text { mientos, instalaciones y equipos. Se proporciona } \\
\text { una intervención temprana a las falencias encon- } \\
\text { tradas en esos factores. }\end{array}$ & $\begin{array}{l}\text { Terapia } \\
\text { Ocupacional }\end{array}$ & $\begin{array}{l}\text { Disminución de incapacidades tempora- } \\
\text { les, eficiencia de los servicios de orienta- } \\
\text { ción en el reintegro y mejor percepción de } \\
\text { la discapacidad por parte del trabajador. }\end{array}$ \\
\hline $\begin{array}{l}\text { Determina la influencia de los subsistemas del } \\
\text { modelo en las características del lugar de trabajo, } \\
\text { la estructura, las exigencias y recursos proporcio- } \\
\text { nados al trabajador para que pueda permanecer } \\
\text { en el trabajo. }\end{array}$ & $\begin{array}{l}\text { Terapia } \\
\text { Ocupacional }\end{array}$ & $\begin{array}{l}\text { Se logran dar indicaciones específicas a } \\
\text { los trabajadores sobre cómo mejorar el } \\
\text { proceso de reincorporación. Orienta los } \\
\text { pasos a seguir en el proceso de mantener } \\
\text { o recuperar el trabajo. }\end{array}$ \\
\hline $\begin{array}{l}\text { Instrumento diseñado para discutir aspectos ocu- } \\
\text { pacionales de la vida del trabajador, identificando } \\
\text { variables psicosociales y ambientales que influyen } \\
\text { en la capacidad de un trabajador para permane- } \\
\text { cer en el trabajo, o retornar a este después de una } \\
\text { lesión o enfermedad. }\end{array}$ & $\begin{array}{l}\text { Terapia } \\
\text { Ocupacional }\end{array}$ & $\begin{array}{l}168 \text { Terapeutas Ocupacionales que labo- } \\
\text { ran en oficinas de servicios públicos de } \\
\text { empleo y centros de atención primaria } \\
\text { reportan que el WRI detecta las dificulta- } \\
\text { des a nivel de interés y motivación por el } \\
\text { trabajo. Favorece la identificación de forta- } \\
\text { lezas y debilidades del trabajador desde el } \\
\text { subsistema volitivo y habituacional. }\end{array}$ \\
\hline $\begin{array}{l}\text { Identifica cualidades del trabajador como: moti- } \\
\text { vación, habilidades físicas, cognitivas y demandas } \\
\text { del lugar, son aspectos relevantes para la prepara- } \\
\text { ción del reintegro laboral. }\end{array}$ & $\begin{array}{l}\text { Terapia } \\
\text { Ocupacional }\end{array}$ & $\begin{array}{l}\text { La evaluación permitió reconocer el nivel } \\
\text { de independencia físico y las habilidades } \\
\text { cognitivas (atención, memoria, juicio) } \\
\text { que requiere el trabajador en el reintegro } \\
\text { laboral. }\end{array}$ \\
\hline
\end{tabular}




\begin{tabular}{|c|c|c|}
\hline Estudios & *Metodología \& Población diana & Estrategia interdisciplinar \\
\hline $\begin{array}{l}\text { McFadden, S., } \\
\text { MacDonald, A., } \\
\text { Fogarty, A., Le, S., \& } \\
\text { Merritt, B. K. (2010) }\end{array}$ & \multirow{3}{*}{$\begin{array}{l}\text { *Revisión de literatura de las pruebas existentes } \\
\text { relacionadas con evaluaciones vocacionales.Bús- } \\
\text { queda en bases de datos como MEDLINE, CINAHL, } \\
\text { Web of Science, Google Scholar y pruebas revisa- } \\
\text { das por pares. }\end{array}$} & $\begin{array}{l}\text { Evaluaciones vocacionales: } \\
\text { Evaluación de la capacidad } \\
\text { funcional (Functional Capacity } \\
\text { Evaluation FCE) }\end{array}$ \\
\hline $\begin{array}{l}\text { McFadden, S., } \\
\text { MacDonald, A., } \\
\text { Fogarty, A., Le, S., \& } \\
\text { Merritt, B. K. (2010) }\end{array}$ & & $\begin{array}{l}\text { Evaluación del Rendimiento del } \\
\text { Trabajo (Assessment of Work } \\
\text { Performance AWP) }\end{array}$ \\
\hline $\begin{array}{l}\text { McFadden, S., } \\
\text { MacDonald, A., } \\
\text { Fogarty, A., Le, S., \& } \\
\text { Merritt, B. K. (2010) }\end{array}$ & & $\begin{array}{l}\text { Evaluación de habilidades motoras } \\
\text { y procesamiento (AMPS). }\end{array}$ \\
\hline
\end{tabular}

Teniendo en cuenta la Tabla No 1, se puede evidenciar que los artículos analizados presentan estrategias interdisciplinarias que explican su aplicación y la importancia de las mismas en el contexto laboral, buscando prepara al usuario para lograr evaluar las habilidades de la persona, las exigencias del cargo, el contexto económico y sociocultural; otras estrategias están más orientadas a evaluar los procesos de adaptación semanas después de que el trabajador se encuentra vinculado nuevamente a sus labores; también existen estrategias en los procesos de seguimiento.

Desde Terapia Ocupacional las estrategias están enfocadas en el reconocimiento de habilidades del trabajador con el fin de generar motivación en el mismo; también se establecen los objetivos del proceso permitiendo un mayor conocimiento en la adecuada ejecución de tareas propias de su puesto de trabajo.

A nivel interdisciplinar, los artículos muestran cómo se pretende indagar sobre la percepción del trabajador con respecto al retorno al trabajo, partiendo de antecedente personales, ambientales y ocupacionales que brinden la información pertinente para la toma de decisiones tanto a nivel individual como organizacional. Asimismo, los artículos presentados hacen énfasis en la ejecución de programas en los cuales se involucra ejercicio físico tales como extensión, flexión y fortalecimiento muscular en trabajadores con diagnósticos de desórdenes musculo-esqueléticos (DME), sin embargo, los resultados arrojados refieren que los mismos son contraproducentes y por consiguiente no presenta datos significativos con respecto al retorno exitoso al trabajo (Snodgrass, 2011).

Uno de los artículos refiere que, a través del condicionamiento operante, se concientiza al trabajador sobre las consecuencias de adoptar comportamientos seguros en la ejecución de sus tareas en el puesto de trabajo, a partir del mismo, se generan graduaciones en la actividad que garantizan el éxito en el retorno laboral. Las intervenciones y capacitaciones durante la jornada laboral también exponen resultados óptimos con respecto al retorno laboral y la reducción de días por incapacidad.

El retorno laboral tradicional en donde no se aplican estrategias para orientar al trabajador, es nombrado por diversos autores quienes concluyen que dicho procedimiento arroja resultados poco significativos con respecto a la disminución del dolor y la reducción en los días de incapacidad.

Como parte de la etapa de seguimiento algunos autores refieren la importancia de realizar visitas al puesto de trabajo, entrevistas y evaluaciones interdisciplinarias logrando identificar factores sociales, ambientales y organizacionales que influyen en la adaptación del trabajador al ámbito laboral y facilitan la implementación de modificaciones pertinentes al caso. 


\begin{tabular}{|l|l|l|}
\hline \multicolumn{1}{|c|}{ Descripción } & Disciplina & \multicolumn{1}{c|}{ Resultados } \\
\hline $\begin{array}{l}\text { Determinan las capacidades físicas de una per- } \\
\text { sona para volver al trabajo, a raíz de una lesión } \\
\text { laboral. }\end{array}$ & $\begin{array}{l}\text { Terapia } \\
\text { Ocupacional }\end{array}$ & $\begin{array}{l}\text { Esta evaluación facilita las modificaciones } \\
\text { en el puesto de trabajo. Se logra obtener } \\
\text { información clara sobre el trabajo físico } \\
\text { (manipulación y manejo de cargas). }\end{array}$ \\
\hline $\begin{array}{l}\text { Evalúa las habilidades para el desempeño de las } \\
\text { actividades de la vida diaria teniendo en cuenta } \\
\text { componentes motores, de proceso y de comuni- } \\
\text { cación e interacción. }\end{array}$ & $\begin{array}{l}\text { Terapia } \\
\text { Ocupacional }\end{array}$ & $\begin{array}{l}\text { Los resultados de la evaluación indican } \\
\text { que la ejecución del trabajador en activi- } \\
\text { dades de la vida diaria puede ser un indi- } \\
\text { cador válido para determinar la capacidad } \\
\text { de volver al trabajo. }\end{array}$ \\
\hline Es un indicador de rendimiento en el trabajo. & $\begin{array}{l}\text { Terapia } \\
\text { Ocupacional }\end{array}$ & $\begin{array}{l}\text { Permite un mayor aprendizaje de tareas } \\
\text { adecuadas en casa y en el trabajo. }\end{array}$ \\
\hline
\end{tabular}

Fuente: Elaboración propia

\section{DISCUSIÓN}

Las personas que no pueden regresar al trabajo experimentan un ajuste psicosocial más pobre, es decir un aumento de la ansiedad y la depresión, aislamiento social y mayores dolencias físicas. Sin embargo, aquellos que retornan al trabajo a través de intervenciones interdisciplinares aumentan la satisfacción de vida, la integración en la comunidad, independencia económica y reducciones en las tasas de discapacidad física (Stergiou-Kita, Yantzi, \& Wan, 2010).

Cabe resaltar que las condiciones en el mercado laboral han cambiado y una parte sustancial de los trabajadores se encuentran enfermos, incluso algunos han perdido su trabajo, ya sea antes de la inclusión o durante el periodo de la intervención. Esto significa que la cantidad de personas que reciben una visita en el lugar de trabajo y son vinculados a un programa de reintegro es reducida. Por tal razón, surge la necesidad de realizar intervenciones interdisciplinarias, donde se deben tener en cuenta actividades como las entrevistas psicológicas, ya que una gran cantidad de trabajadores tienen puntuaciones superiores a 1,5 en varias dimensiones del cuestionario de los 90 síntomas (SCL-90-R), manifestando depresión y ansiedad durante el periodo de evaluación de su desempeño laboral (Fisker, Langberg, Petersen, \& Mortensen, 2013).
Los síntomas depresivos están relacionados con el desempeño laboral en términos de orientación y demanda de la tarea e interacción con el ambiente de trabajo. Estos síntomas afectan de manera significativa la participación en ocupaciones. Un menor nivel de compromiso ocupacional se prevé que afecta en una fase más aguda de la enfermedad o cuando la vida laboral es menos productiva y el proceso de recuperación del trabajador puede tardar más tiempo. Es por esto que, para generar un retorno al trabajo potencial se requiere tener menos síntomas depresivos y mayores niveles de compromiso ocupacional y de empoderamiento, los cuales se logran con la participación de rehabilitación o actividades productivas en el trabajo. Además, los estudios de intervención interdisciplinar de Canadá y los Países Bajos han demostrado un efecto positivo en la reducción del período de licencia por enfermedad en trabajadores (Bejerholm \& Areberg, 2014).

Cabe resaltar que desde Terapia Ocupacional y Psicología proporcionan servicios de formación vocacional, que hoy son un campo central de intervención. Estos profesionales pueden brindar una amplia gama de intervenciones relacionadas con el trabajo, están calificados para evaluar los aspectos físicos, cognitivos, emocionales, sociales y ocupacionales de una persona y las demandas del ambiente de trabajo (Yngve, M., \& Ekbladh, E, 2015). 
Las estrategias utilizadas desde Terapia Ocupacional se basan en el Modelo de Ocupación Humana (MOHO) el cual proporciona información sobre la volición, habituación y capacidad de desempeño, donde se tiene en cuenta el entorno del trabajador y su influencia en el éxito laboral (Bejerholm, U., \& Areberg, C, 2014). Así mismo se puede observar a través del análisis de los artículos que algunas de las estrategias sustentan sus intervenciones a partir de conceptos del modelo de adaptación ocupacional, sin mencionarlo específicamente en estas (McFadden, et al, 2010).

McFadden, et al., 2010, afirman que la Evaluación de la Capacidad Funcional (FCE) no puede captar plenamente la capacidad de un trabajador para realiza tareas laborales. Por lo tanto, es lógico pensar que otras pruebas que evalúan el aspecto psicosocial y el impacto del comportamiento y ambiente en el trabajo se deben combinar con este tipo de evaluación (McFadden, et al 2010); este caso se observa en la Entrevista del Rol de Trabajador (WRI) la cual se puede complementar con I Evaluación del Rendimiento del Trabajo (AWP), diálogo sobre la capacidad para el trabajo (DWA) y Escala de Impacto Ambiental del trabajador (WEIS) (Yngve, M., \& Ekbladh, E, 2015).

\section{CONCLUSIONES}

Es necesario ampliar el concepto de la preparación para el retorno al trabajo, pues la disposición para esta no solo depende del trabajador, sino además de una participación a nivel organizacional y ambiental del puesto de trabajo. Por lo tanto, la disposición para el retorno laboral va más allá de percibir al trabajador preparado para volver, pues se deben proporcionar las condiciones ambientales en el lugar de trabajo para apoyar el retorno laboral de la persona.

En este punto resulta oportuno destacar la importancia del rol de los Terapeutas Ocupacionales en el apoyo a los trabajadores en su regreso al trabajo, sin embargo existen barreras dentro de este proceso relacionadas con la disponibilidad limitada de evaluaciones vocacionales válidas y confiables; cabe mencionar que dichas evaluaciones en su mayoría se centran en las capacidades y funciones del cuerpo, que con frecuencia no cuentan con el impacto psicosocial, conductual y ambiental como factores determinantes del rendimiento y capacidad la boral. Es por esto que con el uso de evaluaciones como la Entrevista del Rol del Trabajador (WRI) los Terapeutas
Ocupacionales evalúan de forma segura y efectiva la capacidad del trabajador para desempeñar tareas (McFadden, et al, 2010).

Fenger y Kramer establecen claramente que se debe trabajar en la planeación de intervenciones de rehabilitación, donde la Entrevista del Rol del Trabajador (WRI) puede ser una medida clínicamente útil para el regreso al trabajo. Además, se considera que la participación de las familias en el proceso de rehabilitación laboral apoya al trabajador en su retorno al trabajo (Fenger \& Kramer, 2007).

En estos procesos de retorno al trabajo es fundamental el apoyo vocacional a trabajadores jóvenes, logrando con esta estrategia plantear metas ocupacionales con el fin de mitigar una situación ocupacional desfavorable. (Bejerholm \& Areberg, 2014).

Finalmente, se identifica la necesidad de generar mayo evidencia sobre el uso de estrategias interdisciplinarias para el retorno al trabajo aplicadas en las diferentes fases del proceso; ya que estas juegan un papel principal en a identificación de factores que afectan directamente la adaptación del trabajador al puesto de trabajo y se generan modificaciones al entorno con el fin de facilitar un retorno exitoso.

\section{REFERENCIAS BIBLIOGRÁFICAS}

Ask, T., \& Magnussen, L. H. (2015). Supervisors' Strategies to Facilitate Work Functioning among Employees with Musculoskeletal Complaints: A Focus Group Study. The Scientific World Journal, 2015.

Bejerholm, U., \& Areberg, C. (2014). Factors related to the return to work potential in persons with severe mental illness. Scandinavian journal of occupational therapy, 21(4), 277-286.

Braathen, T. N., Eftedal, M., Tellnes, G., \& Haugli, L. (2015). Work inclusion self-perceived change in work ability among persons in occupational rehabilitation. Society, Health \& Vulnerability, 6

Boada Mojica, J. D. R., \& Moreno Angarita, M. (2013). Las ARP y el Manua Guía sobre Procedimientos para la Rehabilitación y Reincorporación Ocupacional de los Trabajadores en el Sistema General de Riesgos Profesionales: una aproximación reflexiva Una aproximación reflexiva.

Carroll, C., Rick, J., Pilgrim, H., Cameron, J., \& Hillage, J. (2010). Workplace involvement improves return to work rates among employee with back pain on long-term sick leave: a systematic review of the effectiveness and cost-effectiveness of interventions. Disability and rehabilitation, 32(8), 607-621.

Coutu, M. F, Baril R, Durand, M. J. Co^té, D., \& Rouleau, A. (2007). Representations: an important key to understanding workers' cop- 
ing behaviors during rehabilitation and the return-to-work process. Journal of Occupational Rehabilitation, 17(3), 522-544.

Coutu, M. F., Légaré, F., Durand, M. I., Corbière, M., Stacey, D., Loisel, P., \& Bainbridge, L. (2011). Fostering shared decision making by occupational therapists and workers involved in accidents resulting in persistent musculoskeletal disorders: a study protocol. Implement Sci, 6(22), 1-8.

Désiron, H. A., Donceel, P., de Rijk, A., \& Van Hoof, E. (2013). A conceptual-practice model for occupational therapy to facilitate return to work in breast cancer patients. Journal of occupational rehabilitation, 23(4), 516-526.

Federación de Aseguradores Colombianos FASECOLDA, F. (2015). Datos Riesgos Laborales. Recuperado 09/05/2016 de: http://www.fasecolda.com/index.php/ramos/riesgos-laborales/estadisticas-del-ramo/

Fenger, K., \& Kramer, J. M. (2007). Worker Role Interview: Testing the psychometric properties of the Icelandic version. Scandinavian Journal of Occupational Therapy, 14(3), 160-172.

Fisker, A., Langberg, H., Petersen, T., \& Mortensen, O. S. (2013). Early coordinated multidisciplinary intervention to prevent sickness absence and labour market exclusion in patients with low back pain: study protocol of a randomized controlled trial. BMC musculoskeletal disorders, 14(1), 1 .

Hoefsmit, N., Houkes, I., \& Nijhuis, F. J. (2012). Intervention characteristics that facilitate return to work after sickness absence: a systematic literature review. Journal of occupational rehabilitation, 22(4), 462-477.

Høgelund, J., Holm, A., \& McIntosh, J. (2010). Does graded return-to-work improve sick-listed workers' chance of returning to regular working hours?.Journal of health economics, 29(1), 158-169.

Kielhofher, G. (2011). Modelo de la ocupación humana. Teoría y aplicación. Buenos Aires: Médica Panamericana.

McFadden, S., MacDonald, A., Fogarty, A., Le, S., \& Merritt, B. K. (2010). Vocational assessment: a review of the literature from an occupation-based perspective. Scandinavian Journal of Occupational Therapy, 17(1), 43-48

Ministerio de la protección social. (2010). Manual de procedimientos para la rehabilitación y reincorporación ocupacional de los trabajadores del sistema general de riesgos profesionales. Bogotá.

Netterstrøm, B., \& Bech, P. (2010). Effect of a multidisciplinary stress treatment programme on the return to work rate for persons with work-related stress. A non-randomized controlled study from a stress clinic. BMC public health, 10(1), 658.

OIT, 2005 http://www.ilo.org/global/about-the-ilo/newsroom/news/ WCMS_006102/lang--es/index.htm

Prior, S., Maciver, D., Forsyth, K., Walsh, M., Meiklejohn, A., \& Irvine, L. (2013). Readiness for employment: Perceptions of mental health service users.Community mental health journal, 49(6), 658-667.

Shaw, W. S., Van der Windt, D. A., Main, C. J., Loisel, P., \& Linton, S. J. (2009). Early patient screening and intervention to address individual-level occupational factors ("blue flags") in back disability. Journal of occupational rehabilitation, 19(1), 64-80.
Sirja Eggert MSN, R. N. (2010). Psychosocial factors affecting employees' abilities to return to work. Workplace Health \& Safety, 58(2), 51.

Snodgrass, J. (2011). Effective occupational therapy interventions in the rehabilitation of individuals with work-related low back injuries and illnesses: a systematic review. American Journal of Occupational Therapy, 65(1), 37-43.

Steenstra, I. A., Ibrahim, S. A., Franche, R. L., Hogg-Johnson, S., Shaw, W. S., \& Pransky, G. S. (2010). Validation of a risk factor-based intervention strategy model using data from the readiness for return to work cohort study.Journal of occupational rehabilitation, 20(3), 394-405. Stergiou-Kita, M., Dawson, D. R., \& Rappolt, S. G. (2011). An integrated review of the processes and factors relevant to vocational evaluation following traumatic brain injury. Journal of occupational rehabilitation, 21(3), 374-394.

Stergiou-Kita, M., Yantzi, A., \& Wan, J. (2010). The personal and workplace factors relevant to work readiness evaluation following acquired brain injury: occupational therapists' perceptions. Brain injury, 24(7-8), 948-958.

Tschernetzki-Neilson, P. J., Brintnell, E. S., Haws, C., \& Graham, K. (2007). Changing to an outcome-focused program improves return to work outcomes.Journal of occupational rehabilitation, 17(3), 473-486.

Turner, A., Foster, M., \& Johnson, S. E. (2003). Terapia ocupacional y disfunción física: Principios, Técnicas y práctica. Elsevier España.

Wasiak, R., Young, A. E., Roessler, R. T., McPherson, K. M., Van Poppel, M. N., \& Anema, J. R. (2007). Measuring return to work. Journal of occupational rehabilitation, 17(4), 766-781.

Yngve, M., \& Ekbladh, E. (2015). Clinical utility of the worker role interview: a survey study among Swedish users. Scandinavian journal of occupational therapy, 22(6), 416-42. 